\title{
Prenatal diagnosis of fetal coronary pulmonary artery fistula with pulmonary atresia and ventricular septal defect
}

\author{
Ryo Ishii ${ }^{1}$, Hidekazu Ishida ${ }^{1}$, Fumiko Torigoe ${ }^{1}$, Jun Narita ${ }^{1}$, and Keiichi Ozono ${ }^{1}$ \\ ${ }^{1}$ Osaka University Faculty of Medicine Graduate School of Medicine
}

September 21, 2020

\begin{abstract}
Patients with pulmonary atresia and ventricular septal defect (PA/VSD) demonstrate a wide variety of pulmonary and coronary arteries; additionally, coronary pulmonary artery fistula (CPAF) is a rare manifestation of PA/VSD and is seldom detected during pregnancy. In this report, we present a case of prenatal diagnosis of CPAF in PA/VSD with showing the present impactful images of CPAF in a neonate, which were obtained by using fetal echocardiography and postnatal electrocardiography-gated 320-row computed tomography. Prenatal diagnosis of CPAF can facilitate the provision of better therapeutic strategies after birth.
\end{abstract}

\section{Keywords}

Coronary artery fistula, Tetralogy of Fallot, fetal echocardiography

\section{Introduction}

Patients with pulmonary atresia and ventricular septal defect (PA/VSD) show a variety of pulmonary and coronary artery anatomies [1]. Additionally, a coronary pulmonary artery fistula (CPAF) occurs in $<10 \%$ of patients with PA/VSD [2]. Various types of CPAF associated with PA/VSD have been reported, however, to the best of our knowledge, there is no report of prenatal diagnosis of CPAF with PA/VSD, probably due to the rarity of the condition and the technical difficulty of the imaging method. In this report, we demonstrated clear images of CPAF associated with PA/VSD using fetal echocardiography and confirmed the exact morphology of CPAF in a neonate using electrocardiography (ECG)-gated 320-row computed tomography $(\mathrm{CT})$ in neonate.

\section{Case Report}

The patient's mother was referred to our hospital after being suspected of a fetal cardiac malformation detected with a fetal screening at 24 weeks of gestation. Fetal echocardiography (Voluson E8; GE Healthcare) revealed a perimembranous VSD, $4 \mathrm{~mm}$ in diameter, the aorta riding over the interventricular septum with an overriding rate of $40-50 \%$ on the 5 -chamber view, central pulmonary artery (PA), and major aortopulmonary collateral artery (MAPCA) arising from the descending aorta (dAo). However, the pulmonary valve and pulmonary forward blood flow from the right ventricle were not clearly defined on the right ventricular outflow tract view at 28 weeks of gestation (Fig 1, movie S1A, S1B). Follow-up fetal echocardiography detected a CPAF communicating to the central PA without ductus arteriosus (DA) at 35 weeks of gestation (Fig 2, movie S2). According to these results, we diagnosed CPAF with PA/VSD and talked the possible therapeutic strategies after birth and the potential risk of coronary steal to the parents. Finally, a male infant was delivered via cesarean section at 41 weeks of gestation. On transthoracic echocardiography (TTE) performed immediately after birth, we observed subaortic VSD with the overriding aorta, pulmonary valve atresia, confluent PA, right side aortic arch, and normal right and left coronary sinus without DA, 
which were completely consistent with previous fetal echocardiographic findings. The notable finding was CPAF arising from the proximal RCA connected to the central PA. To observe the appearance of CPAF in detail immediately after birth, cardiac angiography and ECG-gated 320-row CT (Aquilion ONE GENESIS Edition; Toshiba Medical Systems) was performed on day 0 and 3 respectively, and the images of CPAF were consistent with fetal echocardiography findings. CPAF originating from the proximal right coronary artery (RCA) and the right ventricular branch was connected to the central PA. The left coronary artery originated from the left coronary sinus. Two MAPCAs derived from the descending aorta supplied blood to the bilateral lung lobes, indicating a dual supply of pulmonary blood from the central PA and MAPCAs (Fig 3 , movie S3). The patient had tachypnea $(100 / \mathrm{min})$ and required high flow nasal ventilation and nitrogen inhalation therapy from 11 days of age; however, no abnormal electrocardiography findings, such as ST-T alteration, were observed. We performed a central shunt operation (left innominate artery to central PA) using a $3.5 \mathrm{~mm}$ Gore-Tex graft, division of CPAF, and ligation of MAPCAs at 16 days after birth (Fig 4). Thereafter, palliative right ventricular outflow tract reconstruction (RVOTR) using a $12 \mathrm{~mm}$ Gore-Tex conduit with tricuspid valve was performed at five months of age, and intracardiac repair with RVOTR using a $16 \mathrm{~mm}$ Gore-Tex conduit at 21 months of age. The patient demonstrated good growth and neurological development and showed no complications at 36 months of age.

\section{Discussion}

To the best of our knowledge, this is the first case of detection of CPAF with PA/VSD using fetal echocardiography. We present the ECG-gated CT images which confirmed the accuracy of prenatal fetal echocardiography and provided much information that would be useful for surgical treatment. Currently, the evaluation of coronary and pulmonary artery anomalies is essential for surgical planning in patients with PA/VSD. Therefore, high-resolution images of fetal echocardiography and CT play pivotal roles in strategizing therapy after birth. Because prenatal diagnosis of coronary artery anomalies without coronary artery dilation is difficult by fetal echocardiography [3], reports of CPAF with PA/VSD in the fetus are unavailable. It is challenging for sonographers to detect CPAF because its blood flow is too small or with low velocity. For this reason, it may be necessary to control the velocity scale or pulse repetition frequency to diagnose CPAF in the fetus. Furthermore, CT can be used to identify coronary artery anatomy in neonates compared with a significantly higher diagnostic accuracy than TTE and angiography. It provides not only the origin and course of the coronary arteries but also their relationships with the surrounding cardiovascular anatomy [4]. However, a previous study on younger children with lower body weight demonstrated images of the lower resolution of the coronary arteries by CT [5]. In our case, retrospective scanning by a 3-cardiac cycle was performed to visualize the relationship between the coronary artery, CPAF, and mPA at the best motion-free phase, despite the low body weight. In conclusion, careful fetal echocardiography can demonstrate CPAF and the ECG-gated 320-row CT can be a powerful and less-invasive diagnostic modality to make a definitive diagnosis. The accuracy of prenatal diagnosis using fetal echocardiography benefits perinatal counseling and decision-making during planning of therapeutic strategy after birth.

\section{Compliance with Ethical Standards}

Informed consent was obtained from the parents of the patients.

\section{Funding}

None

\section{Conflict of Interest}

The authors declare no conflicts of interest.

\section{Author Contributions}

Ryo Ishii contributed concept/design and drafting article.

Hidekazu Ishida contributed critical revision. 
Fumiko Torigoe contributed data collection.

Jun Narita contributed critical revision.

Keiichi Ozono contributed approval of article.

\section{References}

1) Liao PK, Edwards WD, Julsrud PR, et al: Pulmonary Blood Supply in Patients with Pulmonary Atresia and Ventricular Septal Defect. J Am Coll Cardiol 1985;6:1343-1350.

2) Alkhushi N, Al-Radi OO, Ajlan A, et al: Coronary-Pulmonary Arterial Fistula in a Neonate with Pulmonary Atresia-Ventricular Septal Defect and Single Coronary Artery. Echocardiography 2017;34:1536-1539.

3) Sharland GK, Konta L, Qureshi SA: Prenatal Diagnosis of Isolated Coronary Artery Fistulas: Progression and Outcome in Five Cases. Cardiol Young 2016;26:915-920.

4) Odawara Y, Kawamura Y, Yamasaki Y, et al: Evaluation of Coronary Artery Variations Using Dual-Source Coronary Computed Tomography Angiography in Neonates with Transposition of the Great Arteries. Jpn J Radiol 2019;37:308-314.

5) Tada A, Sato S, Kanie Y et al: Image Quality of Coronary Computed Tomography Angiography with 320-row Area Detector Computed Tomography in Children with Congenital Heart Disease. Pediatr Cardiolol 2016;37:497-503.

\section{Figure Legends}

Fig. 1. ( A) Two-dimensional image of fetal echocardiography showing the 5-chamber view. A perimembranous VSD and aAo overring inter-ventricular septum was seen. (B) Two-dimensional and color doppler image of fetal echocardiography showing three vessels view. Stenotic PA was seen on normal position, but pulmonary valve and pulmonary forward blood flow from the right ventricle was not detected. (C) Twodimensional and color doppler image of fetal echocardiography showing RV outflow view. aAo originated from both RV and LV, but the PA trunk was not detected. MAPCA originating from dAo connected PA without DA.

VSD, ventricular septum defect; aAo, ascending aorta; PA, pulmonary artery; RV, right ventricle; LV, left ventricle; MAPCA, major aortopulmonary collateral artery; DA, ductus arteriosus; dAo, descending aorta.

Fig. 2. (A) Two-dimensional image of fetal echocardiography showing three vessels, tracheal view. Coronary pulmonary artery fistula (CPAF) seems to originate from aAo and communicate with mPA. (B) Color Doppler image of fetal echocardiography showing CPAF supplied blood flow toward mPA. (C) Schema of the fetal echocardiography is shown in Figure 2A and 2B.

CPAF, coronary pulmonary artery fistula; aAo, ascending aorta; mPA, main pulmonary artery

Fig. 3. (A) Aortography shows CPAF originates from RCA and subsequently is connected to the MPA. (B) Electrocardiogram (ECG)-gated 320-row computed tomography angiography (CTA) shows the right coronary artery (RCA) and left coronary artery (LCA) originating from the normal coronary sinus. (C) ECG-gated 320-row CTAespecially shows the positional relationship of RCA and CPAF. RCA and LCA originate from the normal coronary sinus. CPAF originates from the RCA and subsequently is connected to the mPA. The central PA and two MAPCAs supply blood to the same lung field.

RCA, right coronary artery; RVB, right ventricular branch; LCA, left coronary artery; aAo, ascending aorta; dAo, descending aorta; CPAF, coronary pulmonary artery fistula; MAPCA, major aortopulmonary collateral artery; MPA, main pulmonary artery, RPA, right pulmonary artery; LPA, left pulmonary artery.

\section{Fig. 4 .}

The intraoperative view shows the pulmonary artery trunk originates from the right coronary artery (RCA), and the vascular wall of CPAF, which seems to be thinner than that of PA, and likely to be white. 
RCA, right coronary artery; RVB, right ventricular branch; LCA, left coronary artery; aAo, ascending aorta; CPAF, coronary pulmonary artery fistula; MPA, main pulmonary artery; RPA, right pulmonary artery; LPA, left pulmonary artery.

\section{Figure 1}
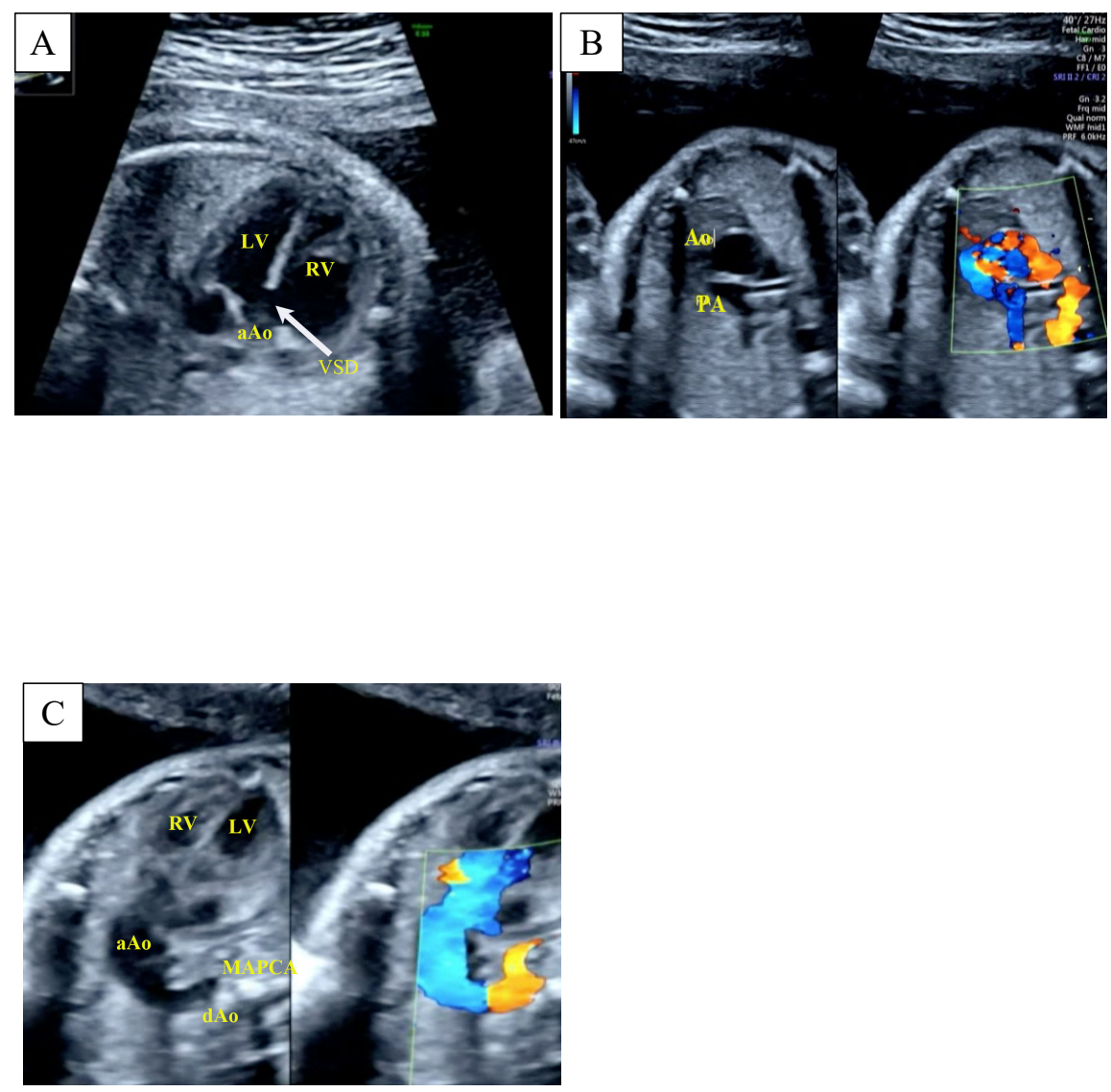
Figure 2
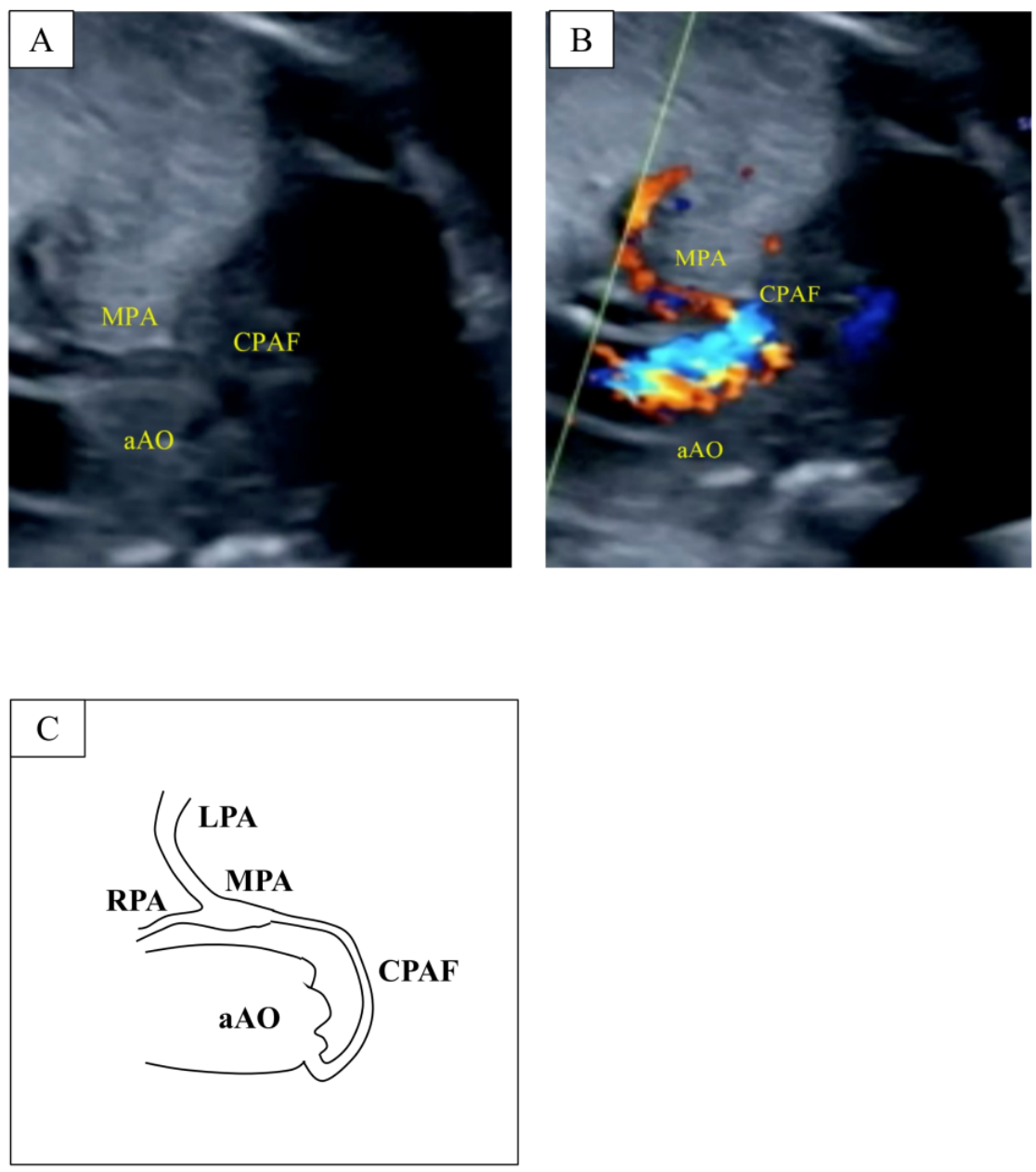
Figure 3
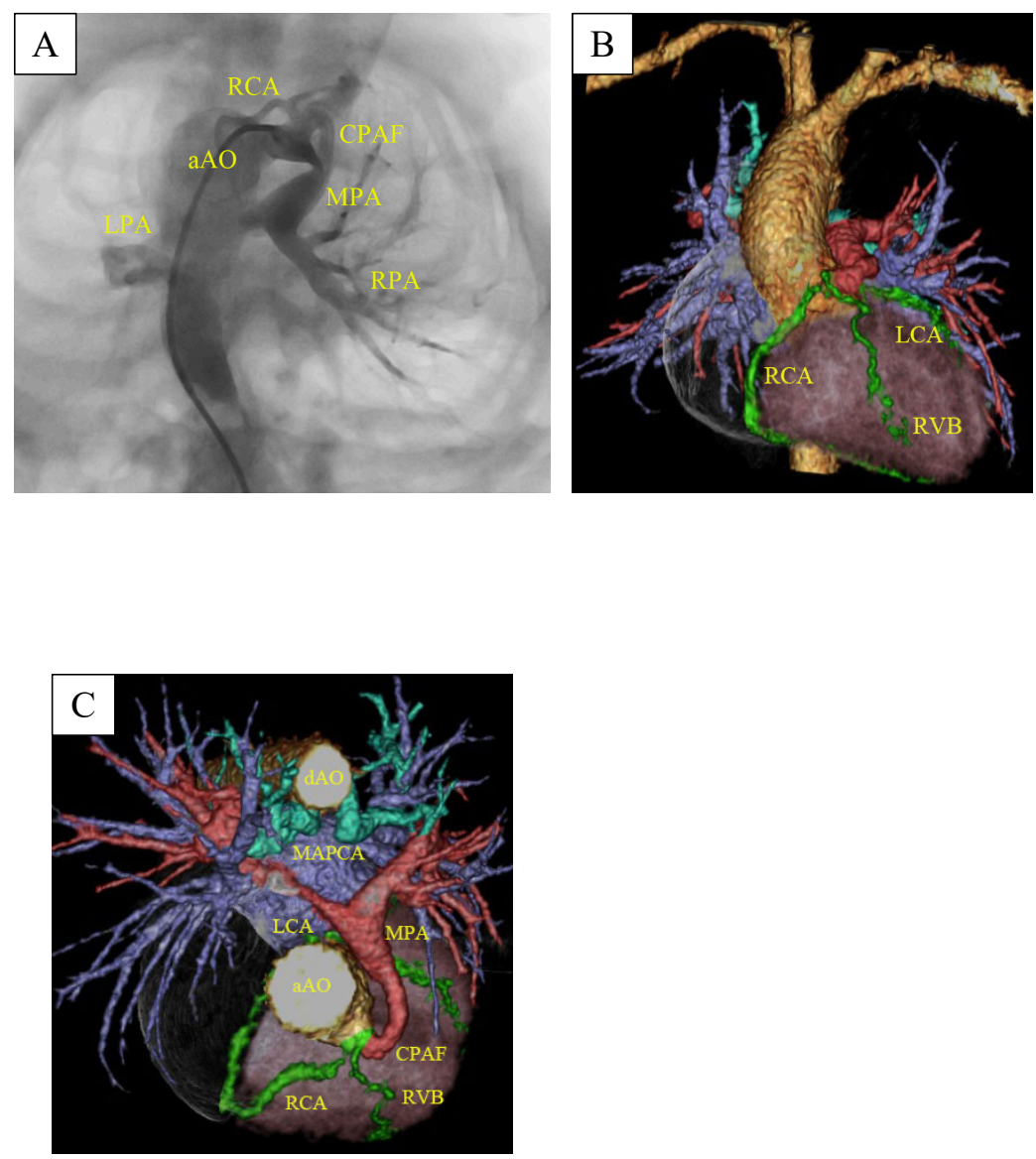
Figure 4

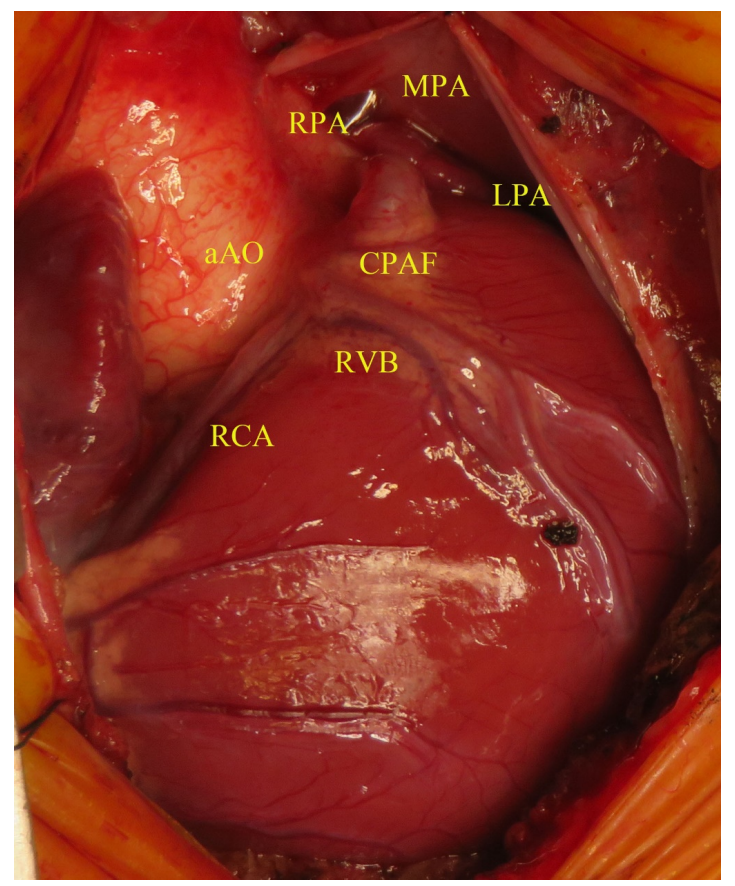

